\title{
A Secure Fuzzy based Enhanced Multi-Queue Job Scheduling Algorithm (EMQJSA) for Load Balancing in Cloud Computing
}

\author{
Aina Sharma \\ Department of Computer Science and Engineering \\ Sri Sai College of Engineering and Technology, \\ Manawala, Amritsar, Punjab, India
}

\author{
B. S. Gill \\ Department of Computer Science and Engineering \\ Sri Sai College of Engineering and Technology, \\ Manawala, Amritsar, Punjab, India
}

\begin{abstract}
The reduction of cost and global utilization of resources shows less wastage of computing power in the era of cloud computing. This field of cloud computing is more preferable now a days because of having most unique and distinct advantages viz. $24 * 7$ on availability mode, no any extra hardware \& software requirement and provides eco-friendly environment. In this research paper, authors proposed a new designed methodology "EMQJSA" which is termed as anEnhanced Multi-Queue Job Scheduling Algorithm whose main purpose is to balance the load among cloud nodes on cloud server by utilizing the concept of cloud job scheduling. The working of this new designed methodology is totally based on Fuzzy logic. The main reason to combine fuzzy logic with newly designed methodology is to achieve high rate of accuracy in terms of results when run cloud jobs on cloud servers. The different parameter considerations viz. waiting time, response time, execution time, trust, throughput and performance plays a vital role in this paper when implemented on newly designed methodology. The main motive of this new designed methodology is to improve the overall performance of the cloud server while performing a managed load balancing. In addition, the other benefit to design this enhanced multi-queue job scheduling algorithm is to provide a safe or secure communication between the cloud user and cloud server. By utilizing this new designed methodology load (cloud heavy jobs) can be easily managed and correspondingly cloud user saves time and the responsibility of cloud resource manager is also reduced or eliminated up to some extent which will ultimately improves the overall performance of the cloud server. Hence, this newly designed proposed methodology named enhanced multi-queue job scheduling algorithm in future will be helpful for producing more accurate and more efficient results.
\end{abstract}

\section{Keywords}

Cloud User, Cloud Server, Cloud Load Balancing Techniques,Cloud Job Scheduling,Multi-Queue Job Scheduling Algorithm, Resource Manager, Cloud Jobs and Time.

\section{INTRODUCTION}

Due to a large number of advantages of cloudcomputing its usage in the market is going to be increases day by day. The main reason to preferring this era of cloud computing is it provides cheap and best service. The main motive of cloud computing is to provide service on demand of the cloud user. In addition, this key technology is also helpful through some other aspects as an example online allotment of computing resources, online storage of user's data at lower cost, availability of data $24 * 7$ on internet facility and most importantly this cloud concept can be used as pay and go approach. [6]In addition, the other main benefit to utilize this cloud computing technologyis it provides a virtual computing environment which is majorly used for business purposes.On the other hand this key technology also show/reflects some drawbacks in terms of critical issues as like security, privacy, load management and fault tolerance etc [7]. These several types of issues can be resolved by applying some techniques and strategies.

In this paper, Authors proposes a new methodology "EMQJSA" which is termed as Enhanced Multi-Queue Job Scheduling Algorithm whose main function is to perform an efficient load balancing during cloud job scheduling. The working of this new designed methodology is based on some certain cloud job scheduling algorithm metrics viz. Waiting Time, response Time, Execution Time, Trust, Throughput and Performance etc.Most importantly, the working of this new designed methodologyis based on Fuzzy Logic. The main significance to utilize fuzzy logic is to achieve more efficient results during cloud job processing rather than existing cloud job scheduling algorithms. The main motive to utilize this new designed methodology is to reduce the workload of cloud resource manager during cloud job scheduling which correspondingly helps to save time of cloud job allocation as well as cloud user time (that is who may be cloud user or cloud service provider). In this way, this newly proposed methodology named Enhanced Multi-Queue Job Scheduling Algorithm clearly outperforms own self in terms of qualitative results.

\section{REVIEW OF LITERATURE}

Inderpal Singh et al (January 2018) [1]: This paper proposing an enhanced multi-queue scheduling method which works on Fuzzy Logic. The main significance to utilize this proposed method on fuzzy logic is to achieve maximum accuracy in results. This enhanced multi-queue scheduling method on fuzzy logic at first grouped the number of jobs according to burst time calculated by its formula. Membership Function plays an important role in enhanced multi-queue job scheduling. It gives more efficient results with decreased execution time as well as overhead. This new designed methodology helps organizations or enterprises to reduce the burden of load balancing when run their applications on cloud.

Inderpal Singh et al (May-June 2015) [2]: This paper has focused on extending the MPQS based scheduling algorithm by using fuzzy values for cloud computing environment. With cloud computing, an organization has the capacity to install computers for the employees and instead of installing applications or application on the individual computers, a single request has the capacity to be loaded on multiple computers. That not only diminishes the software and 
equipment needs of the user's device but also develops the major training of application in to most of the other computers in the system therefore making the user's device a lot more efficient. That workload change has observed many corporations resorting in to cloud computing. The use of fuzzy logic seems to be efficient as it comes up with best alternative for shifting the load from one position to another. Also the use of fuzzy logic will reduce the overall overheads of the live migration techniques.

D.Kannan et al (2017) [3]: In this paper, authors discusses about data security in cloud computing which is more challenging task now a days. So there is a need to protect that data against malicious attacks, unauthorized access, modification or denial of services etc. There is need to ensure the security of cloud data that being exchanged between the users and the cloud. Many security algorithms are available in which mainly cryptography techniques are applied to secure data, but security of all these algorithms can be broken by anyone. Hence the new framework named as fuzzy logic is proposed to secure the data. The main aim of this technique is to provide security from vulnerable attacks in cloud environment. The performance of the proposed method outperforms well with efficient security.

AV.Karthick and E.Ramaraj et al (2014) [4]:This paper shows the efficient job scheduling increases the cloud client satisfaction by proposing a Multi Queue Scheduling (MQS) algorithm for reducing the cost of both reservation and ondemand plans using the global scheduler. The ultimate aim of global scheduler is to share the resources at most the maximum level that's why researchers gives more importance to build a job scheduling algorithms that are further long wellsuited and appropriate for handling the jobs on cloud. The working of proposed methodology is depending on the concept of clustering the jobs based on burst time. During the time of Scheduling the traditional methods such as First Come First Serve, Shortest Job First, EASY, Combinational Backfill and Improved backfill using balance spiral method are creates fragmentation. The proposed method overcome this problem and reduces the starvation with in the process. The proposed MQS method gives more importance to select job dynamically in order to achieve the optimum cloud scheduling problem and hence it utilize the unused free space in an economic way.

Ning Liu and Ziqian Dong et al (2013) [5]:In this paper, Authors present an optimization model fortask scheduling for minimizing energy consumption in cloud computing data centers. The proposed approach was formulated as an integer programming problem to minimize the cloud computing data center energy consumption by scheduling tasks to a minimum numbers of servers while keeping the task response time constraints. Their results shows average task response time and the number of active servers needed to meet such time constraints are bounded through the use of a greedy task scheduling scheme. In addition, we propose the most-efficient server-first task-scheduling scheme to minimize energy expenditure as a practical scheduling scheme. Authors modeled and simulate the proposed scheduling scheme for a data center with heterogeneous tasks. The simulation results show that the proposed task scheduling Scheme reduces server energy consumption on average over 70 times when compared to the energy consumed under a (not-optimized) random-based task-scheduling scheme. We show that energy savings are achieved by minimizing the allocated number of servers.

\section{RESEARCH DESIGN}

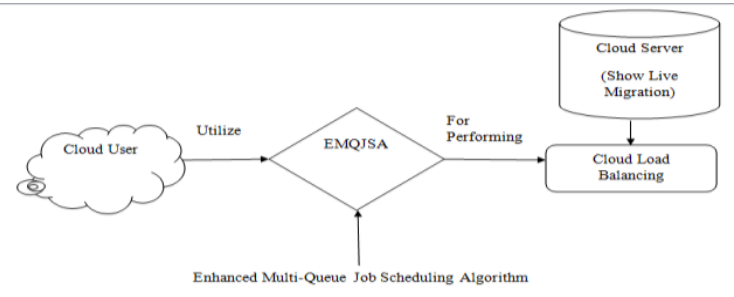

Figure.1:- Interaction between Cloud User and Cloud Server.

4. PROPOSED METHODOLOGY EMQJSA (ENHANCED MULTIQUEUE JOB SCHEDULING ALGORITHM)

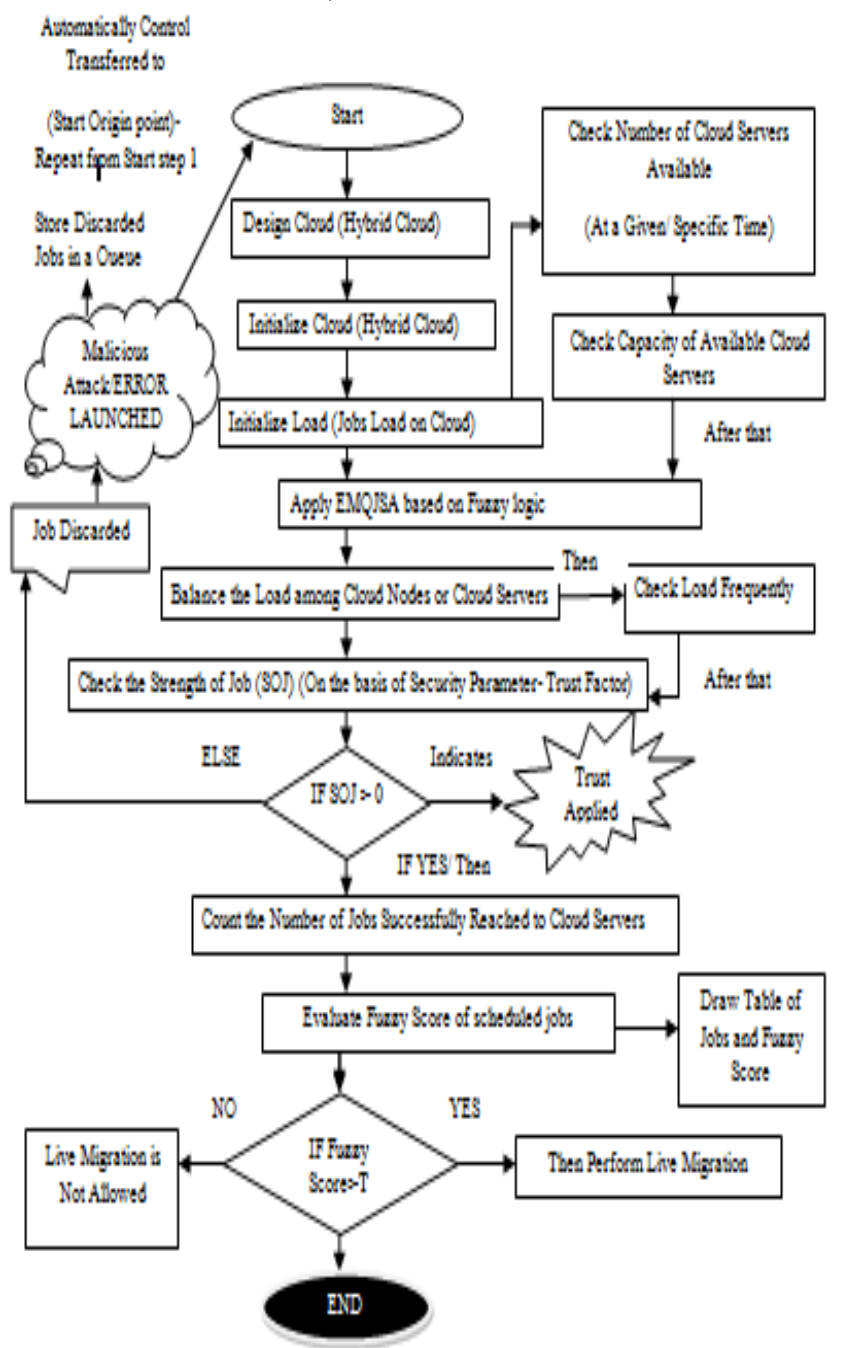

Figure.No.1: Steps Enabled in EMQJSA (Enhanced MultiQueue Job Scheduling Algorithm).

The following steps of proposed methodology EMQJSA are given below: -

Step-1) Initialize Cloud.

Step-2) Initialize load.

Step-3) Apply EMQJSA. 
Step-4) Balance load among cloud nodes.

Step-5) Check the Strength of the Job by Calculating Trust Factor.

Step-6) Check \& Count the number of Nodes Successfully reached to Cloud Server.

Step-7) Evaluate Fuzzy Score of Scheduled Jobs for performing Live Migration.

Step-8) IF FUZZY SCORE > THRESHOULD VALUE, THEN Live Migration of Cloud Servers is Accessible.

Step-9) ELSE FUZY SCORE < THRESHOULD VALUE THEN REPEAT from step 1 to 9. (In this special case, Live migration of Cloud Servers is not possible).

Step-10) End.

\section{CONCLUSIONS}

Different types of cloud job scheduling algorithms are discussed in this research paper. The main significance to study the concept of cloud job scheduling algorithms for efficient cloud job scheduling is to improve the overall performance of the cloud server. Authors designed a new methodology "EMQJSA" which is termed as anEnhanced Multi-Queue Job Scheduling Algorithm whose main purpose is to provide a easy way for performing the task of cloud load balancing ( that is cloud job may be light in weight or heavy in weight) within minimum duration of time. The working of this new designed methodology is based on Multi-Queue Job Scheduling Algorithm. The main motive to design this new methodology is to save resource manager effort of cloud job allotment and save time as well as cost that ultimately improve overall cloud server performance. The utilization of this new designed methodology shows load on cloud nodes can be easily managed and heavy cloud jobs can be easily processed by utilizing the distinct feature of Live Migration which is performed on cloud server.Hence, the different parameter considerations viz. waiting time, response time, execution time, trust, throughput and performance plays a vital role in this paper when implemented on newly designed methodology EMQJSA. In the end, authors say this newly proposed methodology "EMQJSA" clearly outperforms its performance and produces more efficient or accurate results.

\section{REFERENCES}

[1] Inderpal Singh, January 2018. Implementation of Improved Multi-Queue Job Scheduling Algorithm for Load Balancing in Cloud Computing, International Journal of Computer Applications.

[2] Inderpal Singh, May-June 2015. Fuzzy Based Improved Multi Queue Job Scheduling For Cloud Computing, International Journal of Advance Research in Computer Science.

[3]D.Kannan, 2017. A Novel Implementation of Fuzzy Based Secure Cloud System, CIIT-Fuzzy Systems, Open Access Journal.

[4] AV.Karthick and E.Ramaraj, 2014. An Efficient Multi Queue Job Scheduling for Cloud Computing, Published inWorld Congress on Computing and Communication Technologies, IEEE.

[5]Ning Liu and Ziqian Dong, 2013. Task Scheduling and Server Provisioning for Energy-Efficient CloudComputing Data Centers, Published in IEEE 33rd International Conference on Distributed Computing Systems Workshops.

[6]Santosh Shelke \& Utkarsh Urphate, 2017. Cluster Based Approach for Load Balancing Using K-Means in Cloud Computing", International journal of Education and Research.

[7] Sahu, Yatendra, and R. K. Pateriya, 2013. Cloud Computing overview with load balancing techniques, International Journal of Computer Applications. 